\title{
Primary malignant melanoma without melanosis of the colon
}

\author{
Nam Hee Yi, Jang Won Lee, Sang Heon Lee, Ji Hyun Kim, Sam Ryong Jee, Sang Yong Seol \\ Department of Internal Medicine, Busan Paik Hospital, Inje University College of Medicine, Busan, Korea
}

Primary malignant melanoma (PMM) of the gastrointestinal tract is rare. Reported cases of PMM of the lower gastrointestinal tract typically describe anal and rectal involvement rather than colonic lesions. This report describes a rare case of a 50-year-old woman with PMM originating in the colon. The patient presented to Inje University Busan Paik Hospital with a 3-day history of blood-tinged stools. She underwent colonoscopy for a diagnosis of hematochezia. The colonoscopic examination revealed a large-sized semi-pedunculated sigmoid colon polyp with a reddish-colored mucosal surface. Endoscopic mucosal resection was performed, and the final histopathological findings were consistent with a diagnosis of malignant melanoma. Systemic work-up was performed for assessment of metastasis and to identify the primary tumor considering the high metastatic rate of gastrointestinal malignant melanoma; however, no other malignant lesion was detected. Thus, she was diagnosed with colonic PMM. She underwent laparoscopic low anterior resection and lymph node dissection and has been recurrence-free for $>2$ years. (Intest Res 2019;17:561-564)

Key Words: Primary malignant melanoma; Endoscopic mucosal resection; Colon

\section{INTRODUCTION}

Primary malignant melanoma (PMM) rarely affects the GI tract. ${ }^{1}$ The GI tract shows a limited number of melanocytes. GI melanocytes are primarily observed in the anorectum; however, a few melanocytes are also distributed in the colon. Therefore, PMMs may occur in the colon. ${ }^{2}$ Similar to the presentation of other colonic cancers, based on the site of colonic involvement, patients present with various symptoms such as abdominal pain, loss of weight, bleeding, and/or bowel habit change. ${ }^{3}$ Diagnosis of colonic melanoma is confirmed by endoscopic or surgical biopsy. ${ }^{4}$ Curative surgical resection is the treatment of choice; however, adjuvant therapies may be required in a few cases. ${ }^{5}$

Received March 4, 2019. Revised June 13, 2019. Accepted June 14, 2019. Correspondence to Sang Heon Lee, Department of Internal Medicine, Busan Paik Hospital, Inje University College of Medicine, 75 Bokji-ro, Busanjin-gu, Busan 47392, Korea. Tel: +82-51-890-6270, Fax: +82-51-892-0273, E-mail: daschu94@naver.com
We report a case of colonic PMM presenting as a nonpigmented colon polyp. The study was performed in accordance with the principles of the Declaration of Helsinki. Written informed consent was obtained.

\section{CASE REPORT}

A 50-year-old woman presented to Inje University Busan Paik Hospital with a 3-day history of blood-tinged stool. She denied a history of any underlying diseases or an unusual family history, and she was a nonsmoker. Her vital signs were within the normal range, and physical examination was unremarkable.

Initial laboratory investigations revealed the following results: white blood cell count, $4,300 / \mathrm{mm}^{3}$; hemoglobin, $11.8 \mathrm{~g} / \mathrm{dL}$; platelet count, $126,000 / \mathrm{mm}^{3}$, and serum CRP, $0.44 \mathrm{mg} / \mathrm{dL}$.

Colonoscopic examination revealed a $2 \mathrm{~cm}$-sized semi-pedunculated polyp at the distal sigmoid colon, located $20 \mathrm{~cm}$ from the anal verge, with overlying reddish-colored mucosa (Fig. 1). We attempted en bloc resection by snare polypecto- 
my; however, the tumor was eventually removed by piecemeal resection.

Histopathological examination revealed a clear deep resection margin; however, the lateral resection margin could not be confirmed owing to the piecemeal resection. Tumor infiltration depth was pT1, which indicated tumor invasion of the submucosa, without invasion of the muscularis propria. H\&E

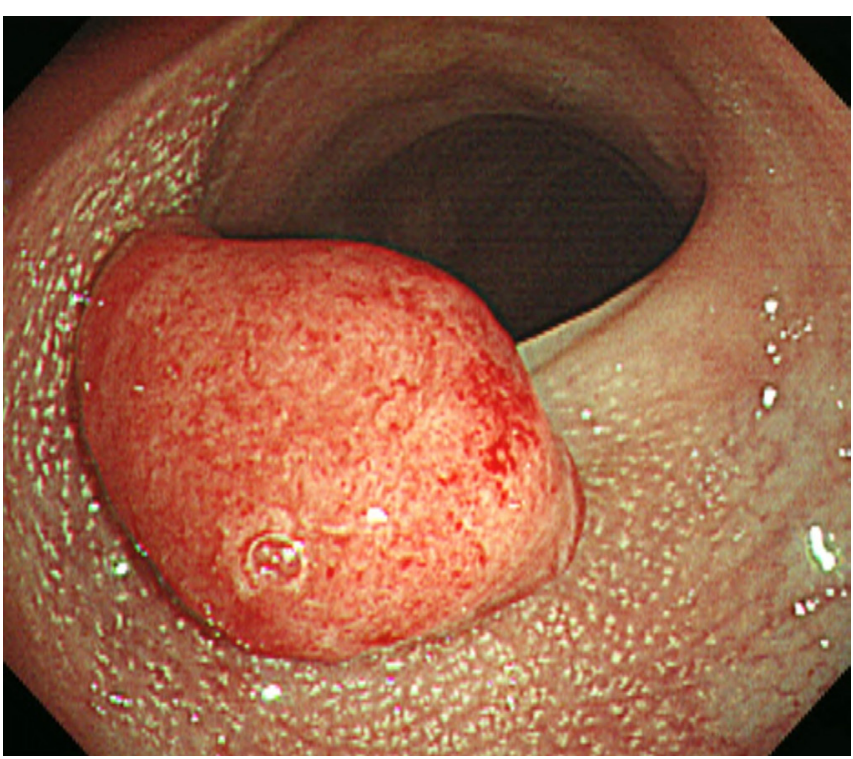

Fig. 1. Colonoscopy finding. A $2 \mathrm{~cm}$-sized reddish polyp was observed at distal sigmoid colon. staining showed an area of spindle-shaped cells and an area of round nodular cells, suggesting sarcomatous and carcinomatous differentiation, respectively (Fig. 2). Small round tumor cells with vesicular nuclei, prominent nucleoli, and multiple mitotic figures were observed in the carcinomatous differentiated area (Fig. 2E).

Immunohistochemical examination revealed negative results for cytokeratin, and positive results for S-100 and HMB45 stains (Fig. 3).

The black pigment, which characterizes melanoma, was not identified; however, the patient was diagnosed with malignant melanoma (MM) based on the aforementioned histopathological findings. Subsequent examinations were performed as follows: (1) The possibility of metastatic MM from the skin was excluded by performing a thorough physical examination. (2) Chest CT and abdominopelvic CT did not reveal any mass or lymph node enlargement in other regions of the body. (3) PETCT did not reveal abnormal ${ }^{18} \mathrm{~F}$-fluorodeoxyglucose uptake. The patient underwent laparoscopy assisted low anterior resection and regional lymph node dissection. No residual melanoma or local lymph node metastasis was identified in the surgically resected specimen.

The patient's progress has been monitored at the aforementioned hospital, and she has been recurrence-free for $>2$ years.
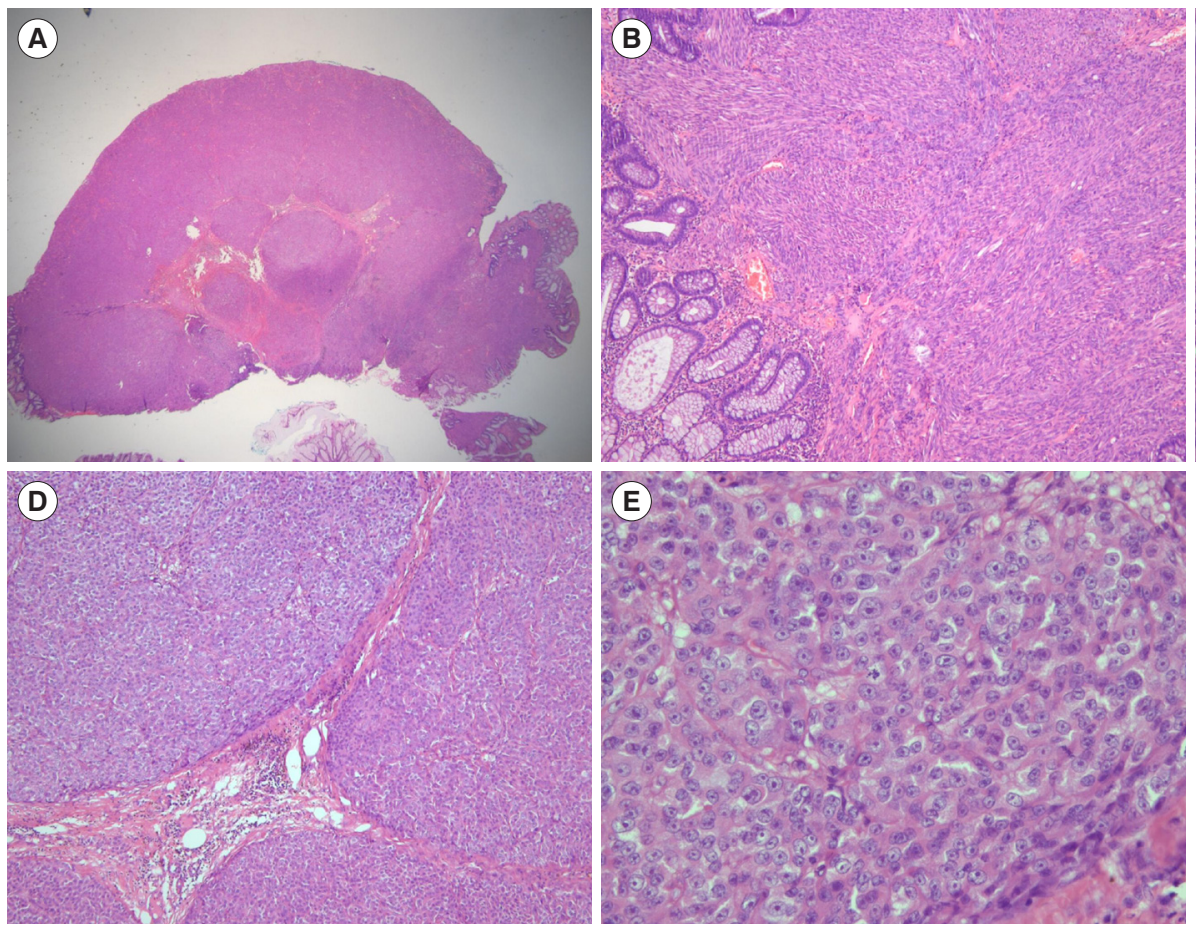

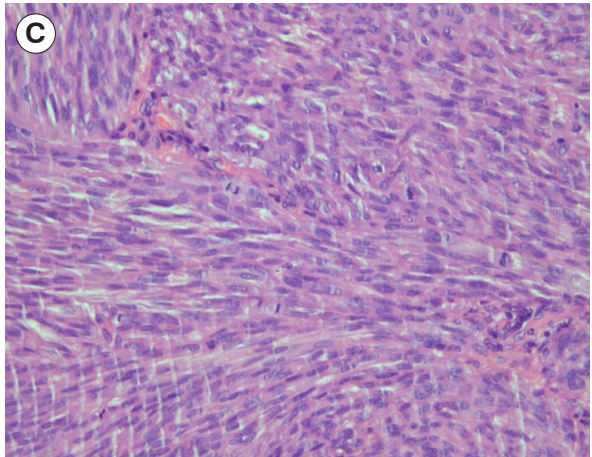

Fig. 2. Pathologic findings. (A) Spindle cellular area and round nodular growing area were observed $\left(H \& E_{1} \times 40\right)$. (B) Spindle cellular area was observed $\left(H \& E_{1} \times 100\right)$. (C) Spindle tumor cells with frequent mitoses were observed $\left(H \& E_{1} \times 400\right)$. (D) Round nodular growing area was observed $\left(H \& E_{1}\right.$ $\times 100$ ). (E) Round tumor cells with vesicular nuclei, prominent nucleoli, and frequent mitoses $(H \& E, \times 400)$. 


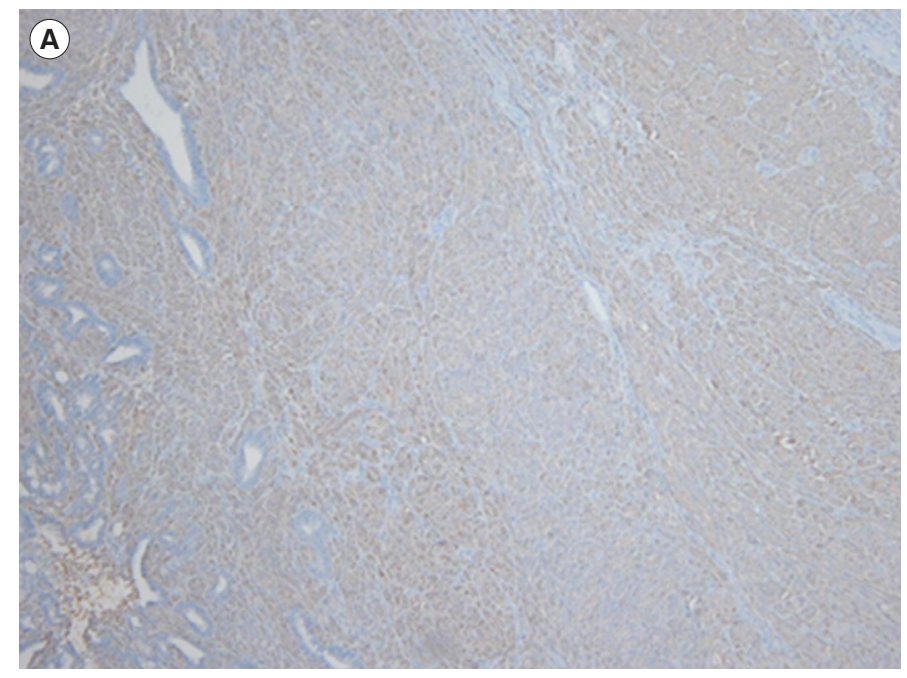

Fig. 3. Immunohistochemistry findings. (A) Positive for S-100 protein.

\section{DISCUSSION}

PMM rarely affects the GI tract. ${ }^{1}$ The GI tract shows a limited number of melanocytes with an even smaller distribution of melanocytes in the colon. Therefore, few cases of colonic PMM have been reported in the literature. ${ }^{2}$ Diagnosis of PMM affecting the GI tract must necessarily include a thorough physical examination to exclude metastatic MM from the skin.

Endoscopic examination of MM involving the GI tract typically reveals a polyp with black pigmentation. Although black pigmentation occurs in most cases, atypical MM without black pigmentation may be observed in some patients. ${ }^{6}$ Therefore, MM could be considered an uncommon colonic malignancy.

On histopathological examination (H\&E stain), the resected specimens reveal melanocytic cells that characterize $\mathrm{PMM}^{7}$ Positive results with HMB-45 stains on immunohistochemical examination confirm the diagnosis, the sensitivity and specificity of the examination being $80 \%$ and $100 \%$, respectively. Additional tests including CT or PET-CT should be performed to assess for distant metastasis. ${ }^{9}$

The treatment of choice is surgical resection with initial assessment to determine tumor resectability. Clinicians should recommend surgical resection for resectable tumors with adjuvant treatment. If curative resection is not possible, secondline treatment such as chemotherapy or targeted immunotherapy may be considered. ${ }^{10}$

Earlier, endoscopic resection was considered for esophageal melanomas confined to the lamina propria of the mucous membrane. However, based on the 7th edition of the TNM manual published by the American Joint Committee on Can-

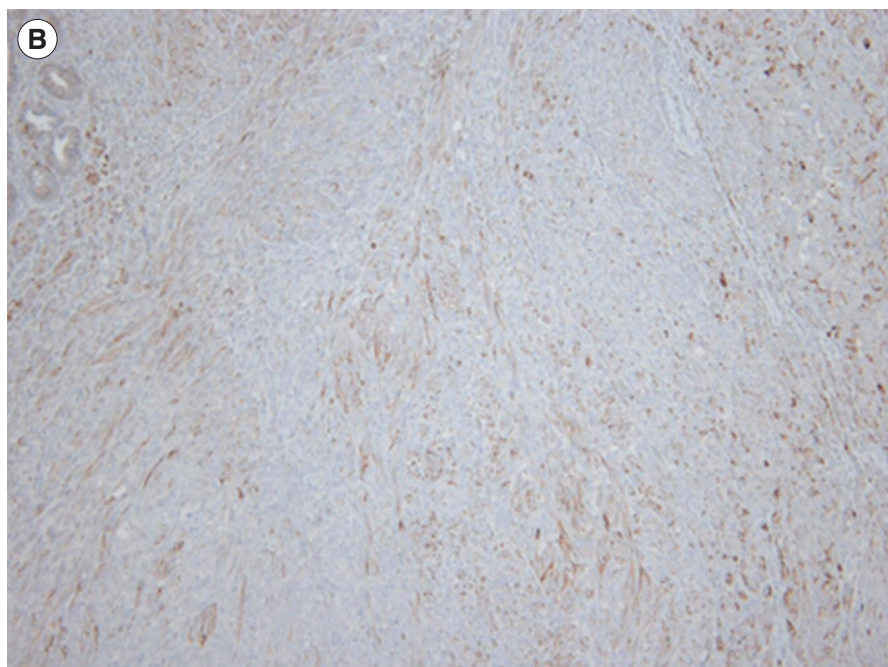

(B) Positive for HMB-45 protein.

cer in 2010, in cases of mucosal melanoma of the upper aerodigestive tract, the $\mathrm{T}$ stage is classified beginning with $\mathrm{T} 3$, omitting the $\mathrm{T} 1$ and $\mathrm{T} 2$ stages. $^{11}$ This indicates that melanomas of the upper aerodigestive tract require more aggressive treatment because these lesions show rapid progression and poor prognosis.

To date, no definitive criteria have been established for colonic PMM. Recently, endoscopic mucosal resection (EMR) was reported in a few cases of PMM of the lower GI tract. ${ }^{12}$ However, considering the typical aggressiveness of MM, radical resection should be considered the first-line treatment, even in lesions showing relatively superficial tissue invasion such as esophageal MM.

In the present case, the endoscopic procedure was performed without a suspicion of melanoma; however, EMR confirmed the diagnosis of $\mathrm{MM}$ of the colon, and the patient underwent radical surgical resection. Follow-up over 2 years postoperatively to monitor disease progression with periodic colonoscopic examinations has not revealed any recurrence.

\section{FINANCIAL SUPPORT}

The authors received no financial support for the research, authorship, and/or publication of this article.

\section{CONFLICT OF INTEREST}

No potential conflict of interest relevant to this article was reported. 


\section{AUTHOR CONTRIBUTION}

Conceptualization: Yi NH, Lee SH. Methodology: Yi NH, Lee SH, Jee SR, Seol SY. Formal analysis: Yi NH, Lee JW. Project administration: Yi NH, Lee SH, Kim JH. Visualization: Yi NH, Lee SH. Writing - original draft: Yi NH, Lee JW. Writing - review and editing: Yi NH, Lee SH, Kim JH. Approval of final manuscript: all authors.

\section{ORCID}

$\begin{array}{ll}\text { Yi NH } & \text { https://orcid.org/0000-0003-0444-6512 } \\ \text { Lee JW } & \text { https://orcid.org/0000-0002-1086-3323 } \\ \text { Lee SH } & \text { https://orcid.org/0000-0001-8210-0889 } \\ \text { Kim JH } & \text { https://orcid.org/0000-0003-0861-2792 } \\ \text { Jee SR } & \text { https://orcid.org/0000-0002-7928-1153 } \\ \text { Seol SY } & \text { https://orcid.org/0000-0003-4525-9916 }\end{array}$

\section{REFERENCES}

1. Houissa F, Bouzaidi S, Mouelhi L, et al. Diffuse primary malignant melanoma of the upper gastrointestinal tract. Gastroenterol Clin Biol 2010;34:85-87.

2. Jacobs-Cohen RJ, Wade PR, Gershon MD. Suppression of the melanogenic potential of migrating neural crest-derived cells by the branchial arches. Anat Rec 2002;268:16-26.

3. Kumar M, DeBono R, Sommerlad BC. Metastatic malignant melanoma of unknown primary site: a case of a possible primary declaring itself 18 months after the secondaries. Br J
Plast Surg 1998;51:258-259.

4. Tessier DJ, McConnell EJ, Young-Fadok T, Wolff BG. Melanoma metastatic to the colon: case series and review of the literature with outcome analysis. Dis Colon Rectum 2003;46:441447.

5. Mandot A, Kazi K, Gupta T, Desai D, Abraham P, Joshi A. Primary malignant melanoma of right colon. Indian J Gastroenterol 2006;25:96-97.

6. Hillenbrand A, Barth TF, Henne-Bruns D, Formentini A. Anorectal amelanotic melanoma. Colorectal Dis 2008;10:612-615.

7. Christova S, Meinhard K, Mihailov I, Alexiev B. Three cases of primary malignant melanoma of the alimentary tract. Gen Diagn Pathol 1996;142:63-67.

8. Gleason BC, Nascimento AF. HMB-45 and Melan-A are useful in the differential diagnosis between granular cell tumor and malignant melanoma. Am J Dermatopathol 2007;29:22-27.

9. Falch C, Stojadinovic A, Hann-von-Weyhern C, et al. Anorectal malignant melanoma: extensive 45-year review and proposal for a novel staging classification. J Am Coll Surg 2013; 217:324-335.

10. Khalid U, Saleem T, Imam AM, Khan MR. Pathogenesis, diagnosis and management of primary melanoma of the colon. World J Surg Oncol 2011;9:14.

11. Balch CM, Gershenwald JE, Soong SJ, et al. Final version of 2009 AJCC melanoma staging and classification. J Clin Oncol 2009;27:6199-6206.

12. Park JH, Lee JR, Yoon HS, et al. Primary anorectal malignant melanoma treated with endoscopic mucosal resection. Intest Res 2015;13:170-174. 